\title{
Platypnea-Orthodeoxia Syndrome Following Resection of an Intrathoracic Extrapulmonary Thymoma
}

\author{
Joseph Kojo Kittah* and Anne Marie Matelski \\ Pulmonology Department, Beloit Memorial Hospital, Wisconsin, USA
}

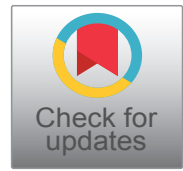

*Corresponding author: Joseph Kojo Kittah, Pulmonology Department, Beloit Memorial Hospital, Wisconsin, USA

\begin{abstract}
We report the case of a 76-year-old female with no significant past medical history who developed Platypnea-orthodeoxia syndrome (POS) following thoracotomy with thymoma resection. Patient underwent right thoracotomy with resection of a $12 \mathrm{~cm}$ right lower lobe mass. The mass was "peeled gently from the pericardium and the aorta", and compressed the right upper, middle, and lower lobes. No lung tissue was resected. Pathology indicated a type B1 thymoma. She was discharged on post-op day \#4 with 4 LPM supplemental oxygen with ambulation.

Two weeks after surgery she was hospitalized with progressive exertional dyspnea and non productive coughing. Oxygen saturation was maintained when supine, but oxygen saturations dropped into the mid 70 s with elevation of the head of the bed, clinching a diagnosis of POS.

Transesophageal echocardiogram revealed a large atrial septal aneurysm with moderate defect by positive bubble study. Cardiac catheterization revealed normal right heart pressures ruling out intracardiac shunting.

She was managed with noninvasive ventilation and eventually discharged to a long-term acute care facility for physical rehabilitation. Atrial septal aneurysm was repaired at a later date with resolution of POS.

There are reports of POS occurring after pneumonectomy; however, in this patient there was no loss in lung volume after surgery. We propose that re-expansion of the lung after resection of a large intrathoracic mass caused V/Q mismatch and initial treatment with positive pressure ventilation proved to be useful in improvement of the orthodeoxia. Patient later received surgical correction of the atrial septal aneurysm.
\end{abstract}

\section{Introduction}

First reported in the literature in 1949, Platypnea-orthodeoxia syndrome (POS) is a rare condition secondary to hepatopulmonary syndrome, cardiac defects causing right-to-left shunting, and intrapulmonary shunting. Patients typically report dyspnea and oxygen desaturation while standing or sitting with an elevated head of the bed. Once supine, these symptoms resolve. There have been several cases of POS following pneumonectomy in patients without elevated cardiac pressures, which typically present approximately 8 weeks after surgery $[1,2]$. With repair of the defect or treatment of the underlying condition, these symptoms abate [1-7].

\section{Case Report}

This patient is a 76-year-old Caucasian female, nonsmoker with no significant past history, seen by her primary care provider for dull, right-sided, positional chest pain. Chest X-ray demonstrated what appeared to be $12 \mathrm{~cm}$ well-circumscribed right lower lobe mass. Subsequent $\mathrm{CT}$ imaging demonstrated a $12 \mathrm{~cm}$ mass with inhomogeneous attenuation and a rind occupying the anterior-inferior portion of the right hemithorax with a small pleural effusion.

Pre-operative pulmonary function tests showed normal spirometry and diffusion capacity with mild air-trapping. PET-CT demonstrated hypermetabolic right lung mass (maximum SUV $=5$ ) and no significant adenopathy. The multidisciplinary team recommended lymph node sampling by bronchoscopy with endobronchial ultrasound-guided transbronchial needle aspiration biopsy (EBUS-TBNA), which was negative for malignancy. Brain MRI was negative for metastatic disease.

The patient had right thoracotomy with resection of mass, right total decortication, and lymphadenectomy.

Citation: Kittah JK, Matelski AM (2020) Platypnea-Orthodeoxia Syndrome Following Resection of an Intrathoracic Extrapulmonary Thymoma. Int J Respir Pulm Med 7:148. doi.org/10.23937/23783516/1410148

Accepted: December 12, 2020; Published: December 14, 2020

Copyright: (c) 2020 Kittah JK, et al. This is an open-access article distributed under the terms of the Creative Commons Attribution License, which permits unrestricted use, distribution, and reproduction in any medium, provided the original author and source are credited 
The mass compressed the right upper, middle, and lower lobes and was "peeled gently from the pericardium and the aorta", with no loss of lung tissue. The patient tolerated the procedure well with no major complications in the perioperative and early postoperative period. Pathology indicated a type B1 thymoma. She discharged on post-operative day \#4 requiring 4 LPM supplemental oxygen on ambulation.

On post-operative day \#12, she presented to the clinic with exertional dyspnea and a nonproductive cough. Her activity had declined over the preceding days. On examination, oxygen saturation was maintained when patient was lying supine, but saturations dropped into the 70s with elevation of the head of the bed. Investigative data including CT angiography was negative. She was readmitted to the ICU.

She was placed on positive airway pressure ventilation (BIPAP) to which she responded. Transesophageal echocardiogram revealed a large atrial septal aneurysm with moderate defect by positive bubble study. Cardiac catheterization revealed normal right heart pressures with no step up or step down defect in the right heart chambers. She was discharged to a long-term acute care facility for physical rehabilitation and she subsequently had repair of the atrial septal aneurysm at a later date at another medical center.

\section{Discussion}

Platypnea-orthodeoxia syndrome (POS) is characterized by dyspnea and oxygen desaturation with upright positioning and symptom resolution in the supine position. Much is still unknown about its physiologic mechanisms [1]. It has been reported as secondary to a hepatopulmonary syndrome or cardiac defect such as patent foramen ovale or atrial septal aneurysm, though there are reports of POS occurring after pneumonectomy [1-5]. In cases of intracardiac defects, an elevation in right-sided heart pressures causes a right-to-left shunt. Pneumonectomy patients have been reported to develop POS in the absence of elevated right heart pressures [1].
Re-expansion of lung after resection of a large intrathoracic mass likely caused $V / Q$ mismatch. We suggest that resultant V/Q mismatch with atrial septal aneurysm triggered the observed clinical syndrome of POS. Our patient did not have significant loss in lung volume after surgery. Managing underlying condition and repair of associated defect is the mainstay of treatment.

\section{Conclusion}

This case illustrates the incidence of POS with thoracotomy without removal of lung tissue. Positive pressure ventilation proved to be useful in initial management of orthodeoxia. Correction of the atrial septal aneurysm was the definitive treatment.

\section{Conflict of Interest}

The authors have no conflict of interest to disclose.

\section{References}

1. Smeenk FW, Postmus PE (1993) Interatrial right-to-left shunting developing after pulmonary resection in the absence of elevated right-sided heart pressures. Review of the literature. Chest 103: 528-531.

2. La Bresh KA, Pietro DA, Coates EO, Khuri SF, Folland ED, et al. (1981) Platypnea syndrome after left pneumonectomy. Chest 79: 605-607.

3. Bhattacharya K, Birla R, Northridge D, Zamvar V (2009) Platypnea-orthodeoxia syndrome: A rare complication after right pneumonectomy. Ann Thorac Surg 88: 2018-2019.

4. Faller $M$, Kessler $R$, Chaouat $A$, Ehrhart $M$, Petit $H$, et al. (2000) Platypnea-orthodeoxia syndrome related to an aortic aneurysm combined with an aneurysm of the atrial septum. Chest 118: 553-537.

5. Marini C, Miniati M, Ambrosino N, Formichi B, Tonelli L, et al. (2006) Dyspnoea and hypoxaemia after lung surgery: The role of interatrial right-to-left shunt. Eur J Respir 28: 174-181.

6. Ptaszek LM, Saldana F, Palacios IF, M Wu S (2009) Platypnea-orthodeoxia syndrome in two previously healthy adults: A case-based review. Clin Med Cardiol 3: 37-43.

7. Robin ED, McCauley RF (1997) An analysis of platypnea-orthodeoxia syndrome including a "new" therapeutic approach. Chest 112: 1449-1451. 